\title{
ANATOMÍA COMPARADA DEL LEÑO DE TRES ESPECIES NATIVAS DE SCHINOPSIS (ANACARDIACEAE)
}

\author{
ANA MARIA GIMENEZ1', FEDERICO CALATAYU², JOSE DIAZ ZIRPOLO³, MARIA EUGENIA \\ FIGUEROA ${ }^{3}$ y DAMIÁN GONZALEZ ${ }^{4}$
}

\begin{abstract}
Resumen: Schinopsis, género endémico de Sudamérica, incluye ocho especies que habitan en los bosques xerófilos chaqueños de Argentina, Bolivia, Paraguay y Brasil. Son cuatro las especies nativas de Argentina, dos de gran importancia económica. El objetivo del trabajo es comparar anatómicamente el xilema de: S. lorentzii (Griseb.) Engl., S. balansae Engl. y Schinopsis marginata Engl. Se trabajó con 5 árboles por especie. Se usó la terminología del Comité de Nomenclatura de IAWA y Tortorelli en las descripciones. Se utilizaron los índices de vulnerabilidad y mesomorfía propuestos por Carlquist para determinar el tipo de comportamiento. Se analizó comparativamente los rasgos anatómicos relevantes. Se analizaron estadísticamente cinco variables cuantitativas: frecuencia de poros $/ \mathrm{mm}^{2}$ (Fv); diámetro tangencial $(\mathrm{Vd})$ y longitud de los elementos vasculares (VI); altura del radio (Rh). El leño homogéneo, presenta diferencias en las variables cuantitativas como frecuencia, diámetro y longitud de vaso. El rasgo anatómico predominante es la presencia de canales secretores de tanino radiales y vasos del duramen ocluidos por tilosis. Los leños de S. lorentzii y S. marginata mostraron gran afinidad y rasgos mesofiticos con tendencia a xerofíticos; mientras que $S$. balansae presenta signos mesofíticos.
\end{abstract}

Palabras clave: Schinopsis, anatomía, xilema, plantas nativas.

Summary: Comparative wood anatomy of tree native species of Schinopsis (Anacardiaceae). Schinopsis, endemic genus of South America, includes eight species that inhabit the Chaco dry forests of Argentina, Bolivia, Paraguay and Brazil. Four species are native to Argentina; two of great economic importance. The aim of this work is to perform comparative wood anatomy of S. lorentzii, S. balansae and S. marginata. It worked with 5 trees of each species. The terminology of IAWA Committee on Nomenclature and Tortorelli were used in wood descriptions. Vulnerability and mesomorphy index proposed by Carlquist, to determine the type of behavior, were used. Relevant anatomical features were comparatively analyzed. Five variables were statistically analyzed: vessel frequency/ $\mathrm{mm}^{2}(\mathrm{Fv})$; tangential vessel diameter $(\mathrm{Vd})$; length of vascular elements (VI) and ray height (Rh). The anatomy of wood is homogeneous, with significant differences in the variables: frequency, diameter and vessel length. Secretory radial tannin channels and tilosis occluding vessel, are predominant anatomical features. Wood anatomy of S. lorentzii and $S$. marginata showed high affinity and mesophitic to xeric trait; while $S$. balansae presents mesophitic signs.

Key words: Schinopsis, anatomy, xylem, native plants.

\section{INTRODUCCIÓN}

Anacardiaceae incluye 73 géneros y 850 especies, de amplia distribución a nivel mundial (Boelcke,

\footnotetext{
1 LAM. Laboratorio de Anatomia de Madera, INSIMA. Facultad de Ciencias Forestales, UNSE. Avda. Belgrano (s) 4200. Santiago del Estero. Argentina, E-mail: amig@ unse.edu.ar.

${ }^{2}$ Ayudante

${ }^{3}$ Becario Doctoral CONICET.

${ }^{4}$ Becario Doctoral FONCYT.
}

1992). El 41\% (39) de sus géneros son nativos del continente americano y el $77 \%$ endémicos. Terrazas (1999) estudia la anatomía de madera de 29 de los 32 géneros de Anacardiaceae distribuidos en América. Destaca que las características que definen el leño de la familia son los canales radiales, las puntuaciones radio vasculares y las fibras septadas. Posee registrado una abundancia de fósiles, en algunas localidades fosilíferas de Argentina (Entre Ríos, Patagonia) desde el Mioceno hasta el Pleistoceno, lo que permite suponer que esta familia ha sido un componente importante 
dentro de las paleo comunidades cenozoicas del sector más austral de América del Sur (Brea, 1999, 2009; Brea \& Franco, 2013).

Los representantes de esta familia son de gran valor económico, ya que producen frutos comestibles, gomas, taninos, tintes y maderas de importancia comercial internacional (Dong \& Baas, 1993). También son conocidas por producir reacciones alérgicas al contacto, debido a la presencia de compuestos fenólicos de tanino producido en los canales radiales del xilema, floema y hojas (Giménez \& Moglia, 2008).

Las especies maderables en general tienen un potencial incalculable para múltiples aplicaciones como ser la producción de vigas, postes, marcos de puertas y ventanas, durmientes, piso, carpintería rural, madera de uso exterior, tornería o por su aptitud carbonífera y leñera (Giménez \& Moglia, 2003).

Tortorelli (1956) menciona a las Anacardiáceas como la familia más importante de la flora dendrológica argentina, debido al valor de numerosas especies de los géneros Astronium, Schinopsis y Schinus. Las especies potencialmente aptas para la extracción de taninos son: Schinopsis quebracho-colorado, S. balansae, Astronium balansae y $A$. urundeuva (Giménez \& Moglia, 1995; Giménez, 2000).

El Gran Chaco Argentino es la región de las Anacardiáceas donde viven 6 géneros y 32 especies, de las cuales 6 son endémicas del género Schinus (Zuloaga et al., 2008).

Schinopsis es endémico de América del Sur e incluye 8 spp. que habitan en los bosques xerófilos del Chaco de Argentina, Bolivia, Paraguay y Brasil (Luna, 2012), de las cuales 4 son nativas de Argentina, dos de gran importancia económica.

Este pequeño género es importante por su influencia cultural y económica, caracterizado por la extrema dureza de su madera (Barberis et al., 2012). La distribución geográfica está restringida a los bosques secos estacionales tropicales y a los subtropicales chaqueños de Sud América (Prado, 2000). Un ancestro de estas especies (Schinopsixylon heckii) es común en sedimentos del Cuaternario en Argentina (Brea, 1999).

Sus especies son explotadas para uso en construcciones, postes, durmientes, y para la obtención de tanino, para numerosas aplicaciones en curtiembres y tratado de cueros, en veterinaria y en actividades pecuarias. Las especies de Schinopsis son dominantes y caracterizan florísticamente los bosques en que habitan. En Argentina habitan 4: S. balansae Engl., S. lorentzii (Griseb.) Engl., S. marginata Engl. y $S$. heterophylla Ragonese \& Castiglioni.

Flores et al. (2013) discuten sobre la identidad taxonómica de $S$. lorentzii y $S$. marginata. Mogni et al. (2014) describen una nueva especie denominada $S$. boqueronensis, endémica del Chaco entre Bolivia y Paraguay, en comparación con $S$. heterophylla.

El leño de Schinopsis ha sido estudiado por Tortorelli (1956), Roth \& Giménez (1997, 2006) y Giménez (1998); otros aspectos analizados fueron su variabilidad radial (Giménez, 2000) y axial (Giménez \& López, 2000, 2002, 2008), el crecimiento de árboles individuales (Giménez \& Ríos, 1999), la estructura cortical (Giménez \& Moglia 2008) y la relación albura y duramen (Giménez et al., 2000). Ferrero \& Villalba (2009) informaron sobre la potencialidad dendrocronológica de $S$. lorentzii para estudios de los bosques secos del chaco sudamericano.

El rasgo distintivo de la familia es la presencia de sustancias de secreción de origen tánico. Los taninos condensados se generan en las capas de floema más externas y activas (Fahn, 1979; Giménez, 2002), la síntesis de dichos compuestos ocurre en el epitelio y en las células parenquimáticas axiales y radiales que rodean a los canales secretores y posiblemente en los vasos, donde las tílides poseen abundantes granos de almidón, los cuales se hidrolizarían en la formación de los compuestos tánicos. Luego este producto es vertido al interior de los largos canales axiales y posteriormente transferidos lentamente a través de los canales radiales al leño comenzando a obstruir los vasos inactivos (Giménez \& Moglia, 1995). De esta manera, estos compuestos son depositados en todo el duramen, incluyendo el lumen de la mayoría de las células parenquimáticas, fibras y vasos.

Se propone en el trabajo realizar la anatomía comparada del xilema de: S. lorentzii (Griseb.) Engl., S. balansae Engl. y S. marginata Engl.

\section{Materiales y Métodos}

El estudio fue realizado con material del bosque 


\section{A. M. Gimenez et al. - Anatomía del leño de especies nativas de Schinopsis}

nativo que se encuentra resguardado en la Xiloteca de LAM (INSIMA), FCF, UNSE (Giménez, Moglia, 2003). Las especies analizadas fueron:

Schinopsis lorentzii (Griseb.) Engl. (quebracho colorado santiagueño). Es la especie leñosa principal de la región Chaqueña Seca. Tiene un potencial incalculable para usos diversos como ser la producción de vigas, postes, durmientes, carpintería de obra en general, tanino, parquet y combustible. Es la principal fuente de materia prima calificada del bosque nativo de la región y su existencia comienza a ser escasa (Giménez \& Moglia, 1993). Las muestras provienen de los Pirpintos, Dpto. Copo, Santiago del Estero.

Schinopsis balansae Engl. (quebracho colorado chaqueño). Especie principal del Chaco húmedo, árbol de gran tamaño, muy apreciado por su madera y por el alto contenido de tanino. Las muestras provienen de Sáenz Peña, Dpto. Comandante Fernández, Chaco.

Schinopsis marginata Engl. (horco quebracho, quebracho del cerro). Es un árbol nativo, característico de los bosques del Chaco Serrano del centro de Argentina, considerado vulnerable debido a la disminución del área de ocupación y los niveles potenciales de explotación (UICN 2011). Las muestras provienen de Guampacha, Dpto. Guasayán, Santiago del Estero.

Se trabajó con 5 ejemplares de cada especie. Las muestras fueron obtenidas de la rodaja a 1,3 m. De cada disco se obtuvo una muestra de $2 \times 2 \mathrm{~cm}$ para realizar los preparados microscópicos de madera. Se tiñeron las muestras con crisoidina-acridina roja y azul de astra, montándose en Entellán. Para los macerados se empleó una solución de una parte de ácido acético glaciar y dos partes de peróxido de hidrógeno (Berlyn \& Misksche, 1976).

En las descripciones del leño se siguió la terminología del Comité de Nomenclatura de IAWA (Baas et al., 1989) y Tortorelli(1956). Las mediciones anatómicas de los caracteres seleccionados se efectuaron siguiendo las recomendaciones de Muñiz \& Coradin (1991), con la medición de 25 elementos por variable e individuo. Las imágenes de microscopía óptica fueron tomadas con video cámara Sony. Además se trabajó con MEB Hitachi TM 1000 Tabletop del Laboratorio de Anatomía de Madera de la UFPR, Curitiba, Brasil.

Se calcularon los índices de vulnerabilidad y mesomorfía propuestos por Carlquist (1977) para determinar el tipo de comportamiento (mesomórfico, xeromórfico):

Índice de Vulnerabilidad $(\mathrm{IV})=($ diámetro medio de vasos/ número de vasos por $\mathrm{mm}^{2}$ ).

Índice de Mesomorfismo $=\mathrm{IV} \times \mathrm{Lev}$

Lev $=$ longitud de elementos vasculares (um)

De acuerdo a los valores de estos índices, los individuos se clasificar en maderas de características mesomórficas (IV > 1; IM > 200) o xeromórficas (IV < 1; IM < 200) (Parra \& Jorge, 2010).

Se aplicaron análisis estadístico multivariados de Componentes Principales (PCA) y de agrupamiento (CA) con el fin de determinar los patrones taxonómicos. Se utilizaron cuatro variables cuantitativas: frecuencia de poros $/ \mathrm{mm}^{2}$ (Fv); diámetro tangencial de los vasos (Vd); longitud de los elementos vasculares (VI); altura de los radios $(\mathrm{Rh})$. Se realizó un análisis no paramétrico de la varianza (ANAVA) para medidas repetidas y la prueba de Kruskal-Wallis $(\alpha=0,05)$ para variables anteriormente citadas. Se utilizó para el análisis estadístico el Programa Profesional InfoStat vs. 2008.

\section{Resultados}

Schinopsis lorentzii (Fig. 1). Árbol de madera muy dura y pesada (peso específico $1,20 \mathrm{~kg} / \mathrm{dm}^{3}$ ), de albura blanco amarillenta y duramen castaño rojizo (Fig. 1 A). Presenta veteado espigado suave, enmascarado por el color, de grano entrelazado y textura fina (Fig. 1B). Con anillos de crecimiento moderadamente demarcados, definido por una banda de 1-3 capas de fibras de paredes gruesas, tipo 3 de Coster (Fig. 1C). El espesor promedio de anillos de crecimiento es de $2.195 \mathrm{~mm}$ (1.6 y 4.5).

El leño se caracteriza por presentar porosidad difusa (Fig. 1D). Los poros son de orientación radial, predominantemente solitarios $(60.3 \%)$, múltiples de 2-3 (39.1\%), multiples largos (1.3\%), geminados y racemiformes: $2.1 \%$. Los miembros de vasos poseen placa de perforación simple (Fig. 1E-F) con las paredes transversales inclinadas. Son elementos pequeños a medianos con diámetro tangencial medio de $73.25 \mu \mathrm{m}$ (60-90), numerosos a muy numerosos con una frecuencia de vasos por $\mathrm{mm}^{2}$ de 14-26. La longitud de los 
Bol. Soc. Argent. Bot. 50 (3) 2015
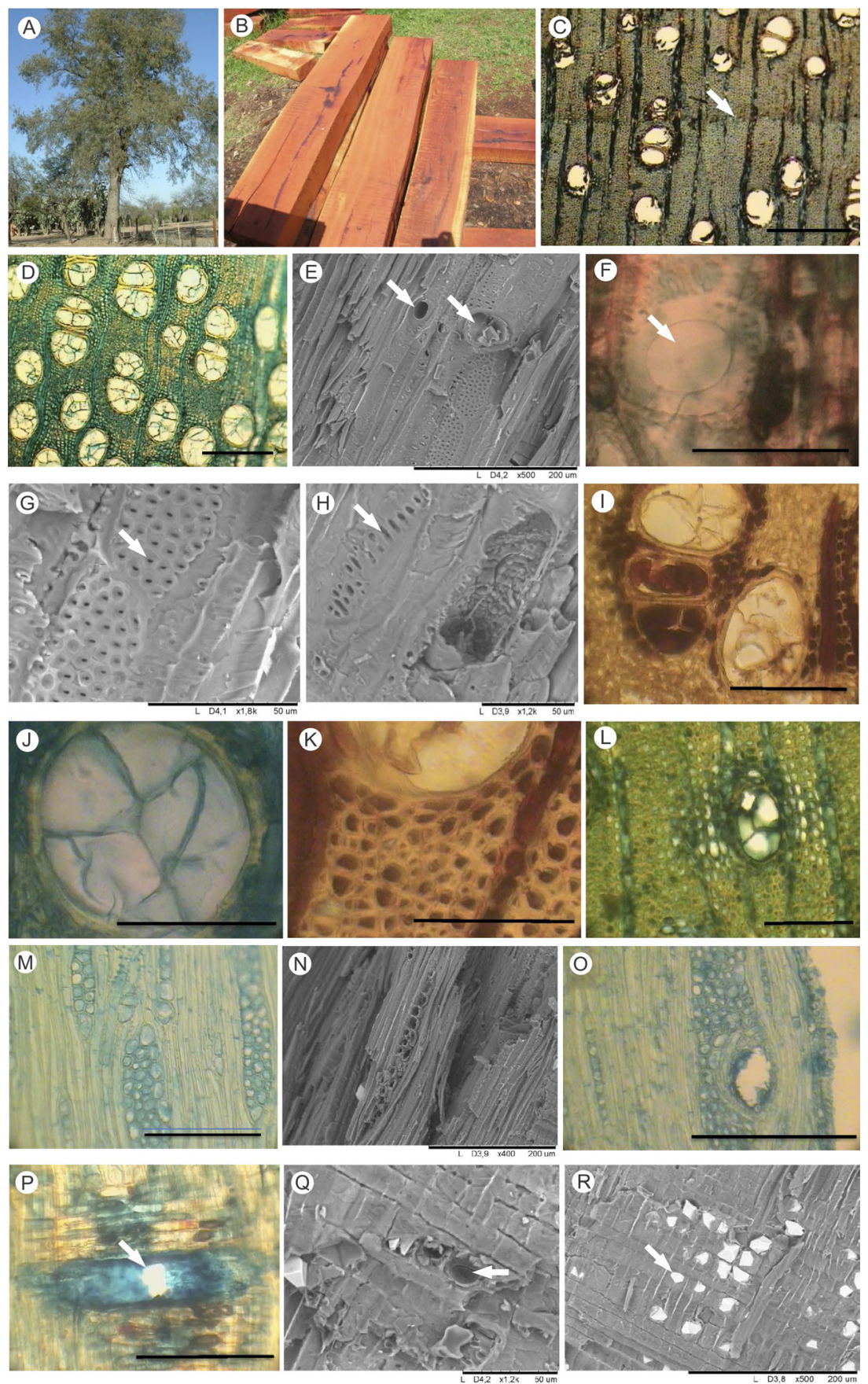

Fig. 1. Schinopsis lorentzii. A: Aspecto del árbol B: Vigas de madera. C: Definición del anillo de crecimiento. D: Leño con porosidad difusa. E-F: Miembros de vaso con placa de perforación simple. G: Puntuaciones intervasculares alternas. $\mathrm{H}$ : Puntuaciones radiovasculares escalariformes. I-J: Vasos del duramen ocluidos por tilosis y tanino. K: Fibra libriforme con tanino en el lumen. L: Parénquima axial paratraqueal vasicéntrico. M-N: Radios leñosos bi y triseriados. O: Radio con canal secretor de tanino. P: Radio con canal en sección radial. Q: Radio con tanino cristalizado. Q: Cristales en células del parénquima radial. La regla de las fotografías con microscopio óptico equivale a $100 \mu \mathrm{m}$. 


\section{A. M. Gimenez et al. - Anatomía del leño de especies nativas de Schinopsis}

miembros de vaso: $272.5 \mu \mathrm{m}$ (150-500).

Las puntuaciones intervasculares (Fig. 1G) son muy numerosas, alternas, algunas coalescentes, con diámetro interno de puntuación $5.6 \mu \mathrm{m}$ (pequeñas). Las puntuaciones radiovasculares son escalariformes (Fig. 1H). En el duramen los vasos están completamente ocluidos por tilosis, tanino y cristales (Fig. 1I-J). El índice de vulnerabilidad (IV) es 3.62, el de mesomorfismo (IM) 985.

El tejido mecánico está constituido por fibras libriformes (Fig. 1K), cortas de valor medio 692,7 $\mu \mathrm{m}$ (489-895). Las puntuaciones simples, diminutas, dispuestas en una hileras, presentan de diámetro externo de un valor medio de 3.1 $\mu \mathrm{m}$, y el espesor medio de pared es de $4.1 \mu \mathrm{m}$, correspondiendo al tipo grueso.

El parénquima axial es de distribución paratraqueal, vasicéntrico delgado formado por 1/3 estratos de células (Fig. 1L). También presenta parénquima axial en banda marginal.

Los radios leñosos son heterocelulares. El cuerpo del radio formado por células procumbentes acompañadas por 1-2 hileras de células verticales dispuestas marginalmente. Los radios presentan células de contacto, son uni a multiseriados (Fig. 1 M-N). El histograma de tipos de radios expresado en $\%$ es: uniseriados (34); biseriados (29); triseriados (23) y multiseriados (14). En estos últimos se observan en su interior canales esquizógenos (Fig. 1O-P). El número promedio de células epiteliales de paredes delgadas que conforman el canal es 13 .

Radios de altura media $268 \mu \mathrm{m}$ (140-400), numerosos $(7-10 / \mathrm{mm})$, se disponen en estructura no estratificada. Se observan abundantes cristales de oxalato de $\mathrm{Ca}$ en el parénquima radial, en las tílides que ocluyen los vasos (Fig. 1Q-R). Hay traqueidas vasculares.

Schinopsis balansae (Fig. 2). De madera muy dura y pesada (Fig. 2A, B, C), peso específico: $1,25 \mathrm{~kg} / \mathrm{dm}^{3}$, de albura blanco rosácea y duramen castaño rojizo. Presenta veteado espigado suave, enmascarado por el color, de grano entrelazado a oblicuo, textura fina, poros poco visibles con 10 aumentos.

Con anillos de crecimiento moderadamente demarcados, de contorno regula, definido a nivel microscópico por una banda delgada de 1-3 capas de fibras de paredes gruesas, tipo 3 de Coster. El espesor promedio de anillos es de $3.54 \mathrm{~mm}$.
El leño se caracteriza por presentar porosidad difusa (Fig. 2D). En el duramen los vasos están completamente ocluidos por tilosis (Fig. 2E), tanino y cristales. Los poros son de orientación radial, múltiples de 2-3 (46\%); solitarios: 45\%, múltiples largos $5.4 \%$ y agrupados/geminados: $4 \%$. Los miembros de vasos poseen placa de perforación simple con las paredes transversales inclinadas (Fig. 2F). Son elementos pequeños a medianos con diámetro tangencial medio promedio de $96 \mu \mathrm{m}$ (50-140), numerosos a muy numerosos con una frecuencia de vasos por $\mathrm{mm}^{2}$ de 11-46.

La longitud de los miembros de vaso promedio es $310 \mu \mathrm{m}$ (200-520). Las puntuaciones intervasculares (Fig. 2G) son muy numerosas, alternas, de forma poligonal, algunas coalescentes. Las puntuaciones radio vasculares horizontales son de bordes reducidos, pero ensanchadas del tipo escalariformes $(5 / 10 \mu \mathrm{m})$ (Fig. $2 \mathrm{H}-\mathrm{I})$. Es frecuente la presencia de tilosis esclerosada (Fig. 2J).

El parénquima axial es de distribución paratraqueal, vasicentrico delgado formado por hasta 3 estratos de células. También presenta parénquima axial en banda marginal.

Los radios leñosos son heterocelulares, el cuerpo formado por células procumbentes con 1-2 hileras de células verticales dispuestas marginalmente. Los radios presentan células de contacto, son uni a multiseriados (Fig. 2K). El histograma de tipos de radios expresado en \% es: uniseriados (20); biseriados (17); triseriados (26) y multiseriados (37). En estos últimos se observan canales esquizógenos de tanino (Fig. 2L). En el interior del canal se encuentra el tanino cristalizado y solidificado, quedando la impronta de las células epiteliales (Fig. 2M). El número promedio de células epiteliales de paredes delgadas que conforman el canal es 16 .

Radios de altura media de $330 \mu \mathrm{m}$ (100-700), muy numerosos $(8 / 12 \mathrm{~mm})$, se disponen en estructura no estratificada.

El tejido mecánico está constituido por fibras libriformes, cortas $649 \mu \mathrm{m}$ (259-838), de paredes gruesas. Las puntuaciones son simples, diminutas y el espesor medio de pared de $4.2 \mu \mathrm{m}$, correspondiendo al tipo grueso. Se observa con MEB un ángulo microfibrilar superior a $40^{\circ}$ (Fig. $2 \mathrm{~N}$ ). Son abundantes los cristales de oxalato de $\mathrm{Ca}$ en el parénquima radial, axial y en las tílides que ocluyen los vasos. Hay traqueidas vasculares. El IV es 6.51 e IM: 2022. 
Bol. Soc. Argent. Bot. 50 (3) 2015
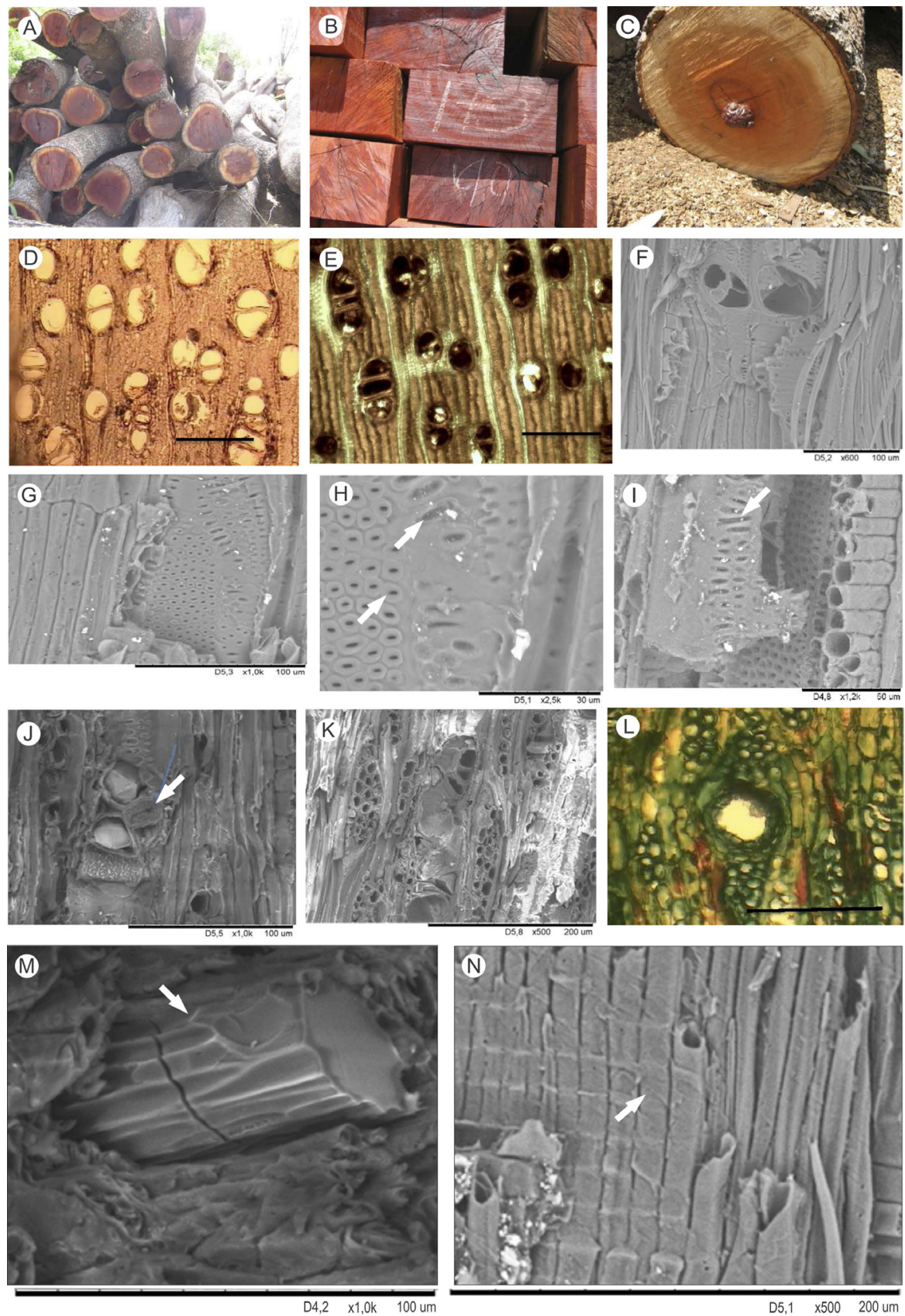

Fig. 2. Schinopsis balansae. A: Rollos de madera en un aserradero. B: Vigas de quebracho. C: Rollo con tanino exudando. D: Leño con porosidad difusa. E: Vasos del duramen ocluidos por tilosis y tanino. F: Miembros de vaso con placa de perforación simple. G: Puntuaciones intervasculares alternas. H-I: Puntuaciones parénquima vasculares escalariformes e intervasculares. J: Miembro de vaso con tilosis esclerosada y cristales. K: Sección tangencial, radios tri y multiseriados. L: Radios con canal secretor de tanino. M: Detalle del canal radial con tanino cristalizado, se observa la impronta de las células epiteliales. $\mathrm{N}$ : Fibras donde se observa el ángulo microfibrilar de la pared celular. La regla de las fotografías con microscopio óptico equivale a $100 \mu \mathrm{m}$. 


\section{A. M. Gimenez et al. - Anatomía del leño de especies nativas de Schinopsis}

Schinopsis marginata (Fig. 3). Árbol de madera muy dura y muy pesada, peso específico: $1,12 \mathrm{~kg} /$ $\mathrm{dm}^{3}$, de albura blanca amarillenta y duramen castaño rojizo. Con un contenido de $12,95 \%$ de taninos, de veteado suave, grano oblicuo a entrelazado (Fig. $3 \mathrm{~A})$.

Anillos de crecimiento son poco demarcados (Fig. 3B), definidos por una banda de fibras engrosadas tangencialmente (1-3 mm. de espesor).

Leño de porosidad difusa (Fig. 3C). Poros en su mayoría solitarios $(45 \%), 41 \%$ de poros múltiples cortos de 2-3, 7.35\% múltiples largos y $6.32 \%$ racemiformes/agrupados. Son poros pequeños (Fig. 3D) con un diámetro tangencial promedio de $66,7 \mu \mathrm{m}$ (20-90), muy numerosos $18-40$ poros por $\mathrm{mm} 2$. En relación a las dos especies anteriores, contiene escasa tilosis. Miembros de vaso con placa de perforación simple (Fig. 3E-F), puntuaciones intervasculares alternas, de márgenes hexagonales (Fig. 3G-H).

Parénquima vertical escaso paratraqueal a vasicéntrico delgado con 2-3 estratos de células. También presenta parénquima axial en banda marginal. Radios heterocelulares (Fig. 3I), fusiformes uni, bi, tri y multiseriados en las siguientes proporciones: $36 \%$ de radios biseriados, $31.7 \%$ de radios uniseriados $27 \%$ de radios triseriados y $2 \%$ de radios multiseriados (Fig. 3J-K-L-M).

La altura de los radios es $229 \mu \mathrm{m}$ (100-420). Por su ancho, se clasifican en moderadamente angostos con dimensiones medias de 39,3 $\mu \mathrm{m}$, entre 14,2 y $71 \mu \mathrm{m}$, de 6-8 radios $/ \mathrm{mm}$. Los radios multiseriados con canales secretores presentan un promedio de células 16.

Fibras cortas $664 \mu \mathrm{m}$ (352-975), de puntuaciones simples y paredes gruesas. Presenta cristales rómbicos en el parénquima radial (Fig. 3O). El IV es 2.83 e IM: 615 .

En Tabla 1 se indican los rasgos distintivos del leño de las 3 especies. Dada la variabilidad de los parámetros que intervienen en el cálculo del IV e IM, se realizó el cálculo con los valores máximos y mínimos de cada uno de ellos, resultando un R2 de 0.97 para una regresión lineal positiva entre ambos índices.

\section{Tabla 1: Caracteres anatómicos descriptivos.}

\begin{tabular}{|c|c|c|c|c|}
\hline Especies & & $\begin{array}{c}\text { Schinopsis } \\
\text { balansae }\end{array}$ & $\begin{array}{c}\text { Schinopsis } \\
\text { lorentzii }\end{array}$ & $\begin{array}{l}\text { Schinopsis } \\
\text { marginata }\end{array}$ \\
\hline \multirow[t]{4}{*}{ Vasos } & Porosidad difusa & * & * & * \\
\hline & Diámetro tangencial de vasos $\mu \mathrm{m}$ & $96(50-140)$ & $73,25(60-90)$ & $66,7(20-90)$ \\
\hline & Frecuencia de poros/mm2 & $14,75(11-46)$ & $20,25(14-26)$ & $20,65(18-40)$ \\
\hline & Longitud de miembro de vaso $\mu \mathrm{m}$ & $310(200-520)$ & $272,5(150-500)$ & $217(130-300)$ \\
\hline \multirow[t]{5}{*}{ Poros \% } & Solitarios & 45 & 60.3 & 45 \\
\hline & Múltiples de 2 - 3 & 46 & 39.1 & 41 \\
\hline & Múltiples largos & 5,4 & 1,3 & 7,35 \\
\hline & Geminados y racemiformnes & 4.0 & 2.1 & 6.32 \\
\hline & Placa de perforación simple & * & * & * \\
\hline Radios & Heterocelular & * & * & * \\
\hline \multirow[t]{7}{*}{ Histograma \% } & Radios uniseriados & 20 & 34 & 31.7 \\
\hline & Radios biseriados & 17 & 29 & 36 \\
\hline & Radios triseriados & 26 & 23 & 27 \\
\hline & Radios multiseriados & 37 & 14 & 2 \\
\hline & Alto de radios $\mu \mathrm{m}$ & $330(100-700)$ & $268(140-400)$ & $229(100-420)$ \\
\hline & $\mathrm{N}^{\circ}$ radios $/ \mathrm{mm}$ & $8 / 12$ & $7 / 10$ & $6 / 8$ \\
\hline & Canales secretores radiales & * & * & * \\
\hline \multirow[t]{2}{*}{ Parénquima axial } & Paratraqueal vasicentrico & * & * & * \\
\hline & en banda marginal & * & * & * \\
\hline Fibras & Longitud $\mu \mathrm{m}$ & $649(259-838)$ & $692,7(895-489)$ & $664(352-975)$ \\
\hline Cristales & & * & * & * \\
\hline IV & Índice de vulnerabilidad & 6,51 & 3,62 & 2,83 \\
\hline IM & Índice de mesomorfía & 2022 & 986 & 615 \\
\hline
\end{tabular}


Bol. Soc. Argent. Bot. 50 (3) 2015
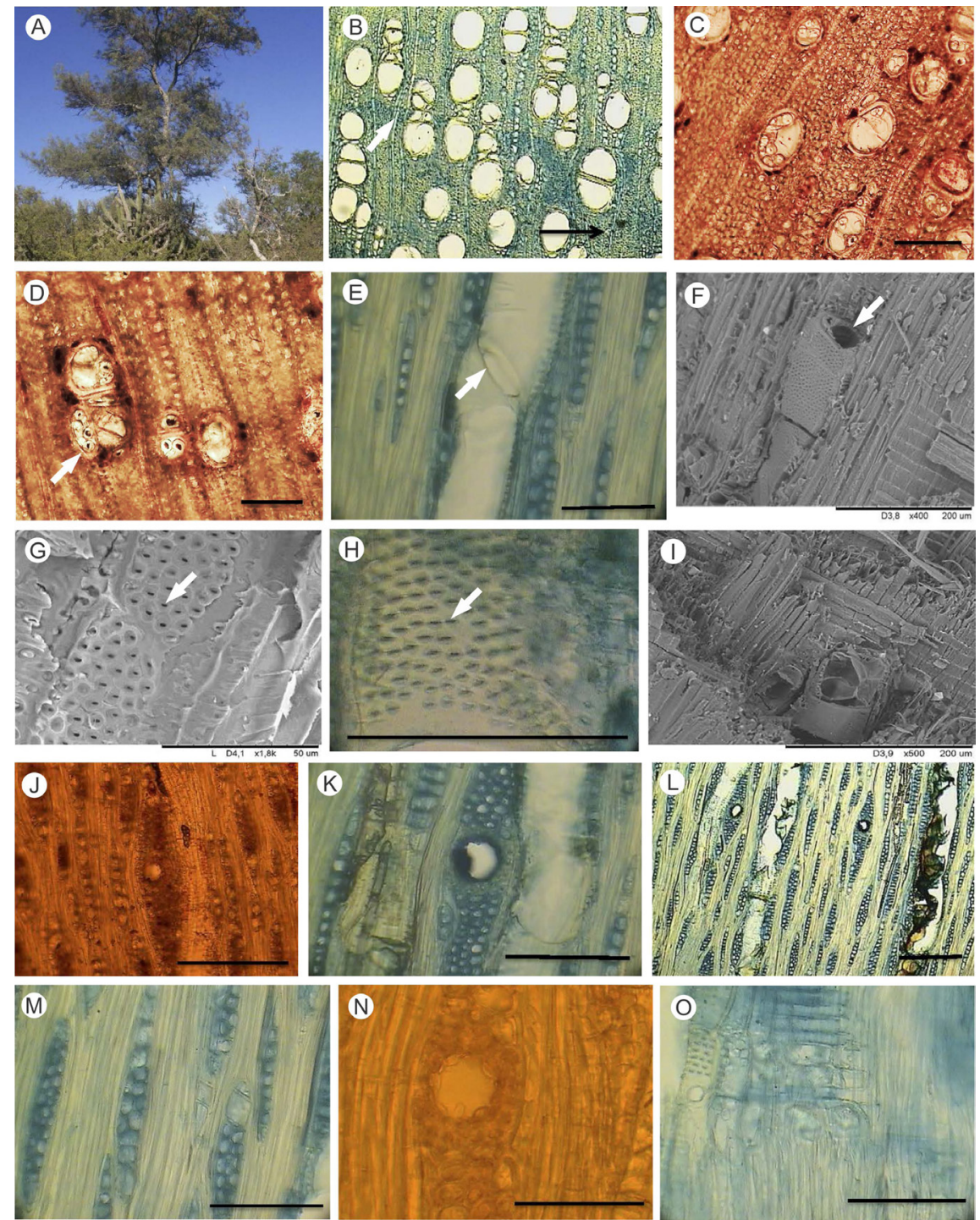

Fig. 3. Schinopsis marginata A: Aspecto del árbol. B: Inicio del anillo de crecimiento. C: Leño de porosidad difusa. D: Vasos de duramen ocluido por tílide y gránulos de almidón. E-F: Miembro de vasos con placa de perforación simple. G-H: Puntuaciones intervasculares alternas, algunas opuestas. I: Sección radial con MEB. J: Sección tangencial radios uni, bi y multiseriados. K: Radio multiseriado con canal secretor. L: Sección tangencial, radios multiseriados con canal secretor de tanino. M: Radios heterocelulares. N: Detalle del canal radial con tanino con células epiteliales de paredes delgadas. O: Cristales en células radiales. La regla de las fotografías con microscopio óptico equivale a $100 \mu \mathrm{m}$.

El análisis multivariado de agrupamiento indica la afinidad anatómica entre $S$. marginata y $S$. lorentzii (Fig. 4).

El análisis multivariado de componentes principales (Fig. 5), explica el $96 \%$ de la variabilidad total en el primer componente. Las variables que mejor expresaron la variabilidad total en el componente 1 son: diámetro de vaso (DV) y longitud de radio (Rl). Para el componente 2 son significativas las variables frecuencia de vasos (FV) y longitud de vasos (VasosL).

En Tabla 2 se indican los resultados del ANAVA 
Promedio (Average linkage)

Distancia: (Euclidea)

3.00

2.00

1.00

$\begin{array}{lllll}0.00 & 0.79 & 1.57 & 2.36 & 3.15\end{array}$

Fig. 4. Análisis de agrupamiento de variables anatómicas cualitativas. 1: S. balansae. 2: $S$. lorentzii, 3: S. marginata.

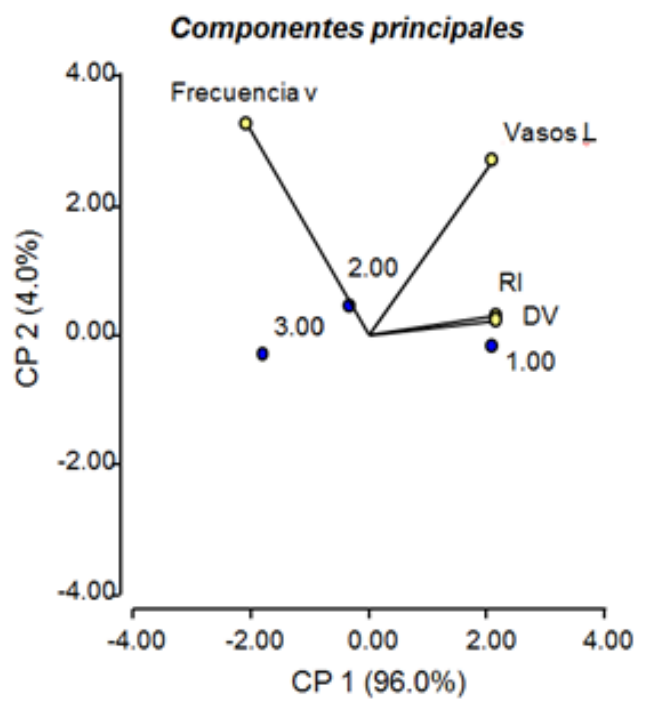

Fig. 5. Análisis multivariado de componentes principales. 1: S. balansae. 2: S. lorentzii, 3: S. marginata.

y test Kruskal-Wallis, donde se pone de manifiesto que existen diferencias significativas entre especies para las variables consideradas. En las Figs. 6 y 7 se establecen los histogramas de tipos de poros y radios por especie.

\section{Discusión}

El xilema de Anacardiaceae (Metcalfe \& Chalk, 1983) se caracteriza por la presencia de sustancias
Radios

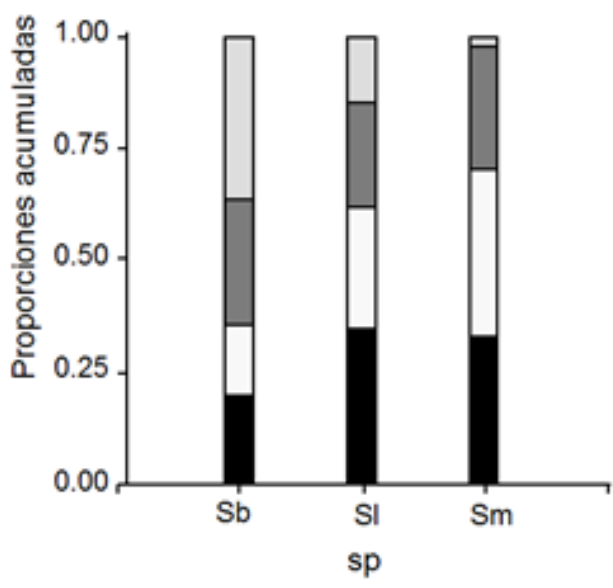

$\square$ uni $\% \square$ bi $\% \square$ tri $\%$
$\square$ tetra $\%$

Fig. 6. Histograma de tipos de radios.

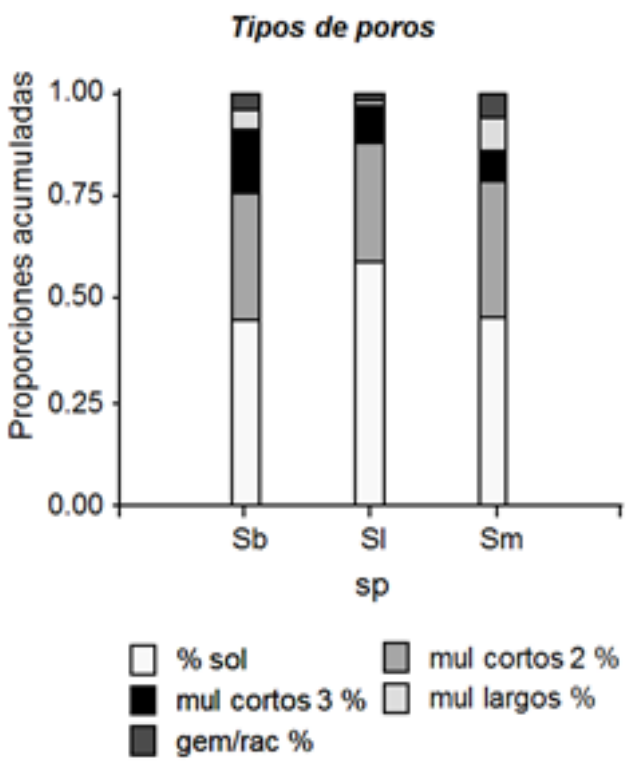

Fig. 7. Histograma de tipos de poros.

tánicas, vasos pequeños a grandes, en casos con patrón ulmoide u oblicuo; placa de perforación simple, excepto en dos géneros, puntuaciones intervasculares alternas, ocasionalmente con porosidad circular y espesamientos espiralados. Es característica la presencia de parénquima paratraqueal escaso, terminal o en distribución 
Tabla 2. ANAVA y test Kruskal-Wallis.

\begin{tabular}{|lcccc|}
\hline \multicolumn{1}{|c}{ Variables anatómicas } & Variación entre sp & S. balansae & S. lorentzii & S. marginata \\
Longitud de vaso & $*$ & B & B & A \\
Diámetro tangencial de vaso & $*$ & B & A & A \\
Frecuencia de vaso & $*$ & A & B & B \\
Alto de radio & ns & B & AB & A \\
\hline
\end{tabular}

Nota: $\mathrm{NS}=$ No significantiva. ${ }^{*}=$ Significantiva $(\alpha=0.05)$.

Letras diferentes indican diferencias significativas con el test de Kruskal-Wallis.

irregular. Predominan los radios 2-3 seriados, en casos de 8-10 seriados en radios con canales horizontales en algunos géneros, usualmente heterogéneos. Fibras con puntuaciones simples, septadas en la mitad de los géneros, de longitud media a muy cortas.

Terrazas (1999) destaca como rasgos relevantes de los géneros americanos la porosidad difusa, frecuencia de vasos mayor a $10 / \mathrm{mm}^{2}$, diámetro de vasos menor a $150 \mu \mathrm{m}$, placa de perforación simple, puntuaciones intervasculares alternas, tílides siempre presentes. Los canales intercelulares son el rasgo típico en el $75 \%$ de los géneros.

Las especies de Schinopsis presentan los rasgos estructurales citados por Tortorelli (1956) y Giménez (2000) para el género. Las 3 especies analizadas son homogéneas en relación a su estructura atómica y pocas las diferencias, solo se manifiestan a nivel de valores promedios y porcentuales en histograma de tipos de poros y radios.

Giménez (1998) indica para S. quebrachocolorado, como características relevantes del leño: tejido mecánico en alta proporción (49.3\%); escaso parénquima axial; poros solitarios fundamentalmente y alto contenido de tanino.

En referencias a los indicadores ecológicos IV e IM: (índices de Vulnerabilidad y Mesomorfismo) (Carlquist, 1988), los valores obtenidos indican el carácter netamente mesomórfico de $S$. balansae y mesomórfico con tendencia al xeromórfico de $S$. lorentzii y $S$. marginata. Ello responde al área de distribución natural y la disponibilidad hídrica de cada especie.

Carlquist (1977) introduce estos índices como indicadores de las relaciones de los caracteres anatómicos del leño y su hábitat. Valores bajos de IV se interpreta como una redundancia de vasos. Ello implica menor posibilidades de desactivación de un determinado número de vasos por efecto del embolismo producido bajo estrés hídrico, que afecte seriamente la conducción en una planta. Miembros de vaso de menor diámetro y mayor frecuencia $/ \mathrm{mm}^{2}$, conforman una estructura que asegura la conducción y evita el embolismo. EL IM presenta valores elevados en especies mesomórficas que habitan en regiones moderadamente húmedas.

La frecuencia de vasos $/ \mathrm{mm}^{2}$ es un indicativo muy importante en referencia al tipo de clima de una región. El número elevado de vasos es frecuente en ambientes secos o fríos, constituyendo una expresión muy sensible del xerofitismo o mesomorfismo (Carlquist, 1988). La frecuencia de vasos es altamente variable en $S$. balansae (CV\%: 37). Ello puede interpretarse como la variación en la disponibilidad de agua que oscila entre las isohietas de 1200 a $800 \mathrm{~mm}$ anuales en su área natural.

Según el histograma de tipos de radios están presentes biseriados, triseriados, uniseriados y multiseriados con canal. En S. balansae predominan los radios multiseriados $(25 \%)$, permitiendo diferenciarse de las otras 2 especies. Tortorelli (1956) indica una proporción de 80\%. En $S$. lorentzii predominan los radios bi y triseriados y en $S$. marginata los radios bi y uniseriados, con escasos tetraseriados. Presentan radios heterogéneos la mayoría de los géneros de la familia (Terrazas, 1999).

Las estructuras de secreción son el rasgo distintivo del género y la familia (Fahn, 1979). Metcalfe \& Chalk (1965) citan la presencia de canales intercelulares en radios de 30 géneros de Anacardiaceae entre ellos, Schinopsis, Astronium, Schinus, Loxopterigium. Terrazas (1999) indica que están presentes en el $60 \%$.

La presencia de canales secretores de terpenos y polisacáridos, característica marcada de 


\section{A. M. Gimenez et al. - Anatomía del leño de especies nativas de Schinopsis}

Anacardiaceae, fue descripto tanto en el cuerpo primario como en el floema secundario (Metcalfe \& Chalk, 1950; Roth \& Giménez, 1997, 2006). Las células epiteliales de pared delgada que constituyen el canal varían de: 13 en $S$. lorentzii; 16 en $S$. balansae y en $S$. marginata: 16.

Sant'ana Santos et al. (2006), indican que los taninos desempeñan un papel importante de defensa contra a herbivoría y contra la invasión de microorganismos patogénicos. Esto es fundamental en la durabilidad natural de la madera, catalogada como de máxima durabilidad (vida útil estimada en más de 30 años) (IRAM, 1998).

El tejido mecánico es distintivo en la calidad de la madera. Las fibras de paredes muy gruesas, están presentes en Astronium, Loxopterigyum, Schinopsis. Se caracteriza en el género por fibras cortas, de paredes muy gruesas, con lumen embebido de tanino y puntuaciones simples pequeñas.

La oclusión total de vasos del duramen por tilosis, hace del leño un material muy especial. La tilosis está formada por células del parénquima axial y radial, que se invaginan a través de una puntuación parénquimo-vascular, bloqueando total o parcialmente el lumen. Se forma en vasos llenos de gas, es decir en elementos no conductivos (Zimmermann, 1983).

Chattaway (1952) analizó el desarrollo de la tilosis y la secreción de gomas en la formación del duramen, estando el proceso condicionado por el tamaño de apertura de las puntuaciones. En las tres especies las puntuaciones tienen un diámetro entre 7-10 $\mu \mathrm{m}$ y apertura de $3 \mu \mathrm{m}$. En una de las muestras se observó tilosis esclerosada, interpretada como una tendencia del xilema a lograr mayor longevidad (Carlquist, 1988). S. balansae y $S$. lorentzii presentan en su duramen oclusión de vasos por tilosis en un $100 \%$.

La tilosis es común en el leño temprano maderas de de porosidad circular, como en Castanea, Fraxinus, Juglans, Ulmus, Robinia, Quercus, etc. También ocurre en maderas de porosidad difusa como Fagus, Populus, Salix (Babos, 1993). Desde un punto de vista evolutivo se considera la tilosis como un rasgo primitivo. En anacardiáceas fósiles se cita la presencia de tilosis (Brea, 1999; Brea \& Franco 2013).

En cuanto a las similitudes de los leños, ya Tortorelli (1956) describe que el leño de S. balansae y $S$. lorenzii son muy difícil de diferenciar. Los valores de variables cuantitativas descriptas por Roth \& Giménez $(1997,2006)$ varían con respecto a los datos expresados en el presente trabajo. Se destaca que el número de muestras con que se trabaja son mayores en este estudio. Se tuvo que recurrir a técnicas estadísticas para diferenciar las especies.

Tortorelli (1956) para la descripción de $S$. quebracho-colorado realizado con 3 ejemplares, indica valores para diámetro de vasos 40-150-200 $\mu \mathrm{m}$; promedio del número de vasos $/ \mathrm{mm}^{2}$ : 8-35. Richter et al. (2000) transcribe los resultados anteriores. El mismo autor para la descripción de $S$. balansae, indica valores para diámetro de vasos 40-150-210 $\mu \mathrm{m}$; promedio del número de vasos/ $\mathrm{mm}^{2}$ : 10-25- 40 .

El análisis de agrupamiento muestra la afinidad anatómica entre $S$. lorentzii y $S$. marginata. Esto de alguna manera, refuerza el trabajo de Flores et al. (2013), que indican no encontrar un límite preciso para 29 caracteres morfológicos estudiados, según el análisis de componentes principales empleado, concluyendo que las variaciones morfológicas están vinculadas al gradiente altitudinal, por lo que incluyen a $S$. marginata bajo la sinonimia de $S$. lorentzii.

En base a lo anteriormente expuesto se concluye que:

El leño de las 3 especies es homogéneo, las diferencias fundamentales están en las variables cuantitativas como frecuencia, diámetro y longitud de vaso. El rasgo anatómico predominante es la presencia de canales radiales secretores de tanino y vasos del duramen ocluidos en su totalidad por tilosis.

A partir de caracteres anatómicos es alta la similitud de $S$. lorentzii y $S$. marginata

$S$. lorentzii y $S$. marginata presentan rasgos mesofíticos con tendencia a xerofíticos y $S$. balansae mesofíticos.

\section{Agradecimientos}

A Fabián Zubrinic, por la compaginación del trabajo, a la Sra. Mirta Sposetti por la realización de los preparados microscópicos. A las Dras. Graciela Bolzón y Silvana Nigosky por el MEB del Laboratorio de Anatomía de Madera de la UFPR, Curitiba, Brasil. 


\section{Biblografía}

BAAS, P., WHEELER, E. \& P. GASSON. 1989. IAWA List of Microscopy features for Hardwood Identification. IAWA Committee.

BABOS, K. 1993. Tyloses formation and the state of health of Quercus petraea trees in Hungary. Inter. Assoc. Wood Anat. J. 14: 239-243.

BARBERIS, I. M., MOGNI, V., OAKLEY, L., ALZUGARAY, C., VESPRINI, J. L. \& PRADO, D. E. 2012. Schinopsis balansae Engl. (Anacardiaceae). Kurtziana 37: 59-86.

BERLYN, G. \& J. MISKSCHE. 1976. Botanical microtechnique and cytochemistry. Iowa State University, Ames.

BOELCKE, O. 1992. Plantas vasculares de la Argentina nativas y exóticas. Ed. Hemisferio Sur, Buenos Aires.

BREA, M. 1999. Leños fósiles de Anacardiaceae y Mimosaceae de la formación El Palmar (Pleistoceno superior) departamento de Concordia, provincia de Entre Ríos, Argentina. Revista Asoc. Paleontol. Argent. 36: 63-69.

BREA, M. \& M. J. FRANCO. 2013. El registro fósil de Anacardiaceae en el Cenozoico Tardío del Noreste argentino. Acta Geol. Lilloana 25: 21-36.

CARLQUIST, S. 1988. Comparative wood anatomy. Springer, Berlin, Heidelberg, New York.

CHATTAWAY, M. M. 1952. The sapwood-heartwood transition. Austr. Forestry 16: 25-34.

DONG, Z. \& P. BAAS. 1993. Wood anatomy of trees and shrubs from China. V. Anacardiaceae. Iawa J. 14: 87-102.

FAHN, A. 1979. Secretory tissues in plants. Academic Press, London.

FERRERO, M. E. \& R. VILLALBA. 2009. Potential of Schinopsis lorentzii for dendrochronological studies in subtropical dry Chaco forests of South America. Trees Struct. Funct. 23: 1275-1284.

FLORES, C., ZAPATER, M.A. \& S. SÜHRING. 2013. Identidad taxonómica de Schinopsis lorentzii y Schinopsis marginata (Anacardiaceae). Darwiniana n.s. 1: 25-38.

GIMÉNEZ, A. M. \& J.G. MOGLIA. 1995. Estructura cortical de Anacardiáceas Argentinas. Quebracho 3: 24-36.

GIMÉNEZ, A. M. 2000. Gradiente radial de los elementos anatómicos del leño en Schinopsis quebracho-colorado (Schlecht.) Barkl. et Meyer, Anacardiaceae. Bosque 21: 37-45.

GIMÉNEZ, A. M. \& J. G. MOGLIA. 2003. Arboles del Chaco Argentino. Guía para el reconocimiento dendrológico. Facultad de Ciencias Forestales, UNSE, Santiago del Estero.

GIMÉNEZ, A. M. 2002. Cambios estructurales producidos durante el desarrollo de la corteza en Schinopsis quebracho-colorado (Schlecht.) Barkl. et Meyer, Anacardiaceae. Quebracho 9: 60-70.

GIMÉNEZ, A. M. \& C. R. LÓPEZ. 2002. Variación longitudinal de los elementos del leño en Schinopsis quebracho-colorado (Schlecht.) Barkl. et Meyer Anacardiaceae. Madera y Bosques 8: 27-38.

GIMENEZ, A. M. \& C. R. LOPEZ. 2008. Caracteres anatómicos que determinan la variabilidad del leño en Schinopsis quebracho-colorado (Schlecht.) Barkl. et Meyer, Anacardiaceae. Forest Systems 9: 219-235.

GIMENEZ, A. M. \& MOGLIA, G. 2008. Estructura cortical de las anacardiáceas argentinas. Forest Systems 4: 189-203.

GIMÉNEZ, A. M., RÍOS, N. \& J. G. MOGLIA, 2000. Relación albura-duramen en tres especies arbóreas de la Región Chaqueña Seca. Quebracho 8: 56-63.

GIMÉNEZ, A.M. \& C. R. LÓPEZ. 2000. Caracteres anatómicos que determinan la variabilidad del leño en Schinopsis quebracho-colorado (Schlecht.) Barkl. et Meyer, Anacardiaceae. Forest Systems 9: 219-235.

GIMÉNEZ, A.M. \& N. RÍOS. 1999. Crecimiento de Schinopsis quebracho-colorado, Anacardiaceae. Madera y Bosques 5: 35-51.

GIMÉNEZ, A.M. 1998. Influencia de la edad sobre los caracteres anatómicos y el crecimiento de Schinopsis quebracho-colorado, Anacardiaceae. Tesis Doctoral, Facultad de Agronomía y Zootecnia, Universidad Nacional de Tucumán.

GIMÉNEZ, A.M. 2000. Gradiente radial de los elementos anatómicos del leño en Schinopsis quebrachocolorado (Schlecht.) Barkl. et Meyer, Anacardiaceae. Bosque (Valdivia) 21: 37-45.

IRAM. 1998. Norma: Preservación de maderas. Maderas preservadas mediante procesos con presión en autoclave. Norma 9600.

IUCN. 2011. Red List of threatened species. World Conservation Monitoring Centre.

LUNA, C. V. 2012. Distribución e importancia maderera de la familia Anacardiaceae en el gran Chaco Argentino. Ra Ximhai 8: 83-95.

METCALFE, C. \& L. CHALK. 1983. Anatomy of the dicotyledons. 2nd Ed. Vol. II. Wood structure and conclusion of the general introduction. Claredon Press, Oxford.

MOGNI, V. Y., OAKLEY, L. J., JIMÉNEZ, M. V. \& D. E PRADO. 2014. A new tree species of Schinopsis (Anacardiaceae) from Paraguay and Bolivia. Phytotaxa 175: 141-147.

MUÑIZ, G. \& V. CORADIN. 1991. Norma de procedimientos en estudios de anatomía da madeira. II Gimnospermae. ABNT, Laboratorio de Produtos Florestais, Serie Técnica, Brasilia. 


\section{A. M. Gimenez et al. - Anatomía del leño de especies nativas de Schinopsis}

PARRA, M. \& S. JORGE. 2010 Determinación de índices de vulnerabilidad y Mesomorfía en especies de laurales de la selva San Eusebio (Mérida, Venezuela). Pittieria 34: 13-22.

PRADO, D.E. 2000. Seasonally dry forests of tropical South America: from forgotten ecosystems to a new phytogeographic unit. Edinburgh J. Bot. 57: 437-461.

RAGONESE, A. \& J. CASTIGLIONI. 1947. Nueva especie del género Schinopsis y área geográfica de las especies argentinas. Revista Invest. Agric. 1: 93-100.

RICHTER, H.G. \& M.J. DALLWITZ. 2000 onwards. Commercial timbers: descriptions, illustrations, identification, and information retrieval. Version: 25th June 2009. http://delta-intkey.com.

ROTH, I. \& A. M. GIMÉNEZ de BOLZÓN. 1997. Argentine Chaco forests: dendrology, tree structure and economic use. 1. The semi-arid Chaco. Encyclopedia of plant anatomy. Borntraeger, Berlin, Stuttgart.

ROTH, I. \& A. M. GIMÉNEZ. 2006. Argentine Chaco forests. Dendrology, tree structure, and economic use. 2. The humid Chaco. Encyclopedia of plant anatomy. Borntraeger, Berlin, Stuttgart.
SANT'ANNA-SANTOS, B., THADEO, M., MEIRA, R., STROZI ALVES, M. \& L. ASCENSAO. 2006. Anatomia e histoquímica das estruturas secretoras do caule de Spondias dulcis Forst. F. (Anacardiaceae). Árvore 30: 481-489.

TERRAZAS, T. 1999. Anatomía de la madera de Anacardiaceae con énfasis en los géneros americanos. Bol. Soc. Bot. México 64: 103-109.

TORTORELLI, L. 1956. Maderas y bosques argentinos. $1^{\circ}$ ed. Ed. ACME, Buenos Aires.

ZIMMERMANN, M. H. 1983. Xylem structure and the ascent of sap. Springer, Berlin, Heildergerg .

ZULOAGA, F. O., O. MORRONE \& M. J. BELGRANO. 2008. Anacardiaceae. En F. O. ZULOAGA, O. MORRONE \& M. J. BELGRANO (eds.), Catálogo de las plantas vasculares del cono sur. Monogr. Syst. Bot. Missouri Bot. Gard. 107: 1043-1052.

Recibido el 11 de diciembre de 2014, aceptado el 22 de mayo de 2015. 
